\title{
STABILITY-BASED LIVE MIGRATION USING DYNAMIC MARGIN
}

\author{
Ryu Sakamoto ${ }^{1}$, Ryuichi Takahashi ${ }^{2}$ and Yoshiaki Fukazawa ${ }^{1}$ \\ ${ }^{1}$ Waseda University, Japan \\ ${ }^{2}$ Ibaraki University, Japan
}

\begin{abstract}
Virtualized environments are common in cloud services. In a virtualized environment, the efficiency of resources is improved by overcommitting. However, excessive overcommits may overload a virtual machine (VM) and subsequently affect other VMs. Hence, methods that predict the load are used to appropriately place VMs. The load of each VM is typically predicted by using the CPU utilization, but this rate can fluctuate greatly. Consequently, a certain margin is common in the predicted value. However, if VMs have greater variations than expected, overload is possible. If a load is predicted to exceed the capacity, some VMs in the host should migrate to other hosts with more room. The live migration technique is used for migrations while running applications. However, if the margin setting is inaccurate, live migrations will be inefficient.

In this paper, we propose a method to perform a live migration using dynamic margin setting. This method gives larger margins to VMs with large fluctuations and smaller margins to VMs with small fluctuations. This method also allows more appropriate predictions to be performed when the fluctuations in the CPU usage is large. Additionally, for stable VMs, resource efficiency may be increased by setting a small margin. In the experiments, this method shows better results than static margin setting, and it is expected to reduce SLA (Service Level Agreement) violations and improve resource utilization efficiency when used for live migrations.
\end{abstract}

\section{KEYWORDS}

Migration, Prediction, Cloud

\section{INTRODUCTION}

Due to the active development of web services, numerous types of web services exist. The load of web services changes with time, the number of users, etc. If the load exceeds the server resources providing the service, the service may fall into a "down" state. In such a state, the service becomes inaccessible to current and new users, negatively impacting all users as well as the service provider.

In this situation, it is difficult to instantly increase the resources of on-premises services to address the down state. Waiting is the only solution. On the other hand, if a web service uses cloud services, resources can be instantaneously increased and a down state can be resolved quickly. Furthermore, when the load is small, it is possible to reduce resources and running costs for cloud services.

One advantage of cloud services compared to on-premises services is that the required performance can be used on demand. Another is that the operating costs are lower. For these reasons, cloud services are common in recent web services. Cloud services are being used in diverse situations.

Consider the cloud service provider side. In a cloud service data center, resource virtualization is actively performed. Since overcommits can be performed by virtualization, the utilization efficiency of physical resources is increased. Overcommitting means that virtual resources are allocated such that the capacity of the actual physical resources is exceeded. If the target of overcommit is a CPU, multiple virtual CPUs may be allocated to one physical CPU at present [Openstack2018]. This is because there are not many situations where all virtual machines (VMs) use all allocated resources. However, if excessive overcommits are present or some VMs' loads increase simultaneously, the sum of all loads may exceed the physical resources of the host machine, causing the host to become overloaded. Overloading affects other VMs that are allocated on the same host machine. To prevent this situation, some of the VMs that are allocated to one host machine need to be moved to another host machine. However, this process requires that the VM is shut down, moved, and restarted. 
Shutting down is not easy because the applications on the VM stop. Fortunately, this issue can be overcome by using live migration technology.

Live migration is a technology that allows transfer to another host machine without shutting down the VM. For example, in a data center, this is realized by managing the VM placement using a management agent, which monitors resource utilization. Host machine overload is detected or predicted by numerical values such as the resource utilization based on the real-time load situation or prediction. Then VMs are moved to another host machine. This prevents occurrence of SLA violations. In a traditional migration of a VM often requires the VM to be shut down. However, downtime can be eliminated by live migration technology.

Live migration is a very valuable technique, but it involves a time overhead. For example, if a live migration is performed after the host machine is overloaded, the performance will decrease until the migration is complete. There are frequent situations where a live migration is required when applications with a fluctuating load like web services are running on a VM. A previous study tried to decrease the number of SLA violations by predicting the future load from past CPU utilization data and doing live migration for situations where the load greatly changes [Farahnakian2013]. By predicting the CPU utilization, which VM should be migrated and which host machine is likely to be overloaded can be visualized, enabling efficient live migration. In previous studies, migration is done when the sum of the predicted values or the measured values of the CPU utilization of VMs exceeds a threshold (for example, 95\% of the physical resources). However, CPU utilization constantly fluctuates, and the variation depends on many factors such as the characteristics of the running software [Abrahao2004]. Therefore, CPU utilization is predicted by giving a certain margin. On an unstable VM, its margin may be violated and the host machine's resources may be insufficient. This leads to host machine overload, affecting not only the VM itself but also other VMs on the host. Conversely, if a fixed value margin is also given to a stable VM, which is in an idle state, the margin is excessive and resources are not used efficiently. Moreover, a live migration should consider the load fluctuation characteristics for each VM because the degree of fluctuation varies between host machines. These situations may result in the need for frequent migrations.

In response to these problems, this paper proposes "stability" to realize a more efficient live migration. Here stability means a metric representing the degree of fluctuation in the CPU utilization. This makes it possible to set different margins for unstable VMs and stable VMs and to migrate more appropriately. This approach should reduce the number of SLA violations, etc. Briefly, first we calculate the stability by using the predicted CPU utilization and the measured value. Then we dynamically set the margin based on these, and perform live migrations.

\section{PROBLEMS WITH THE CONVENTIONAL METHOD}

As mentioned earlier, the conventional method calculates the margin by the same method for all VMs, regardless of the actual CPU utilization. Thus, the same value is also assigned to a CPU with $0 \%$ utilization.

The CPU utilization of an unstable VM may deviate significantly from the predicted value. Then the CPU utilization may exceed the set margin. If there are some unstable VMs in the same host machine, the CPU utilization of the host machine itself may become too high, causing an SLA violation because the required performance by the cloud service cannot be provided. From the viewpoint of a cloud service user, it becomes impossible to use the cloud service with confidence. To mitigate a loss of confidence, cloud services must use many VMs or a larger VM, increasing the costs. Consequently, failure to properly set the margin leads to unnecessary use of resources and costs for both cloud service providers and users.

\section{RELATED WORKS}

In addition to using CPU utilization, some methods predict resource utilization for network resources and memory resources [Farahnakian2013] [Hieu2015]. Load prediction algorithms employ a linear regression [Farahnakian2013] as a function to predict resource utilization. In another approach, which is not a function approximation, the state change of the load is predicted and detected by the Markov model to determine when to perform a live migration [Melhem2017]. 
Because live migration technology used as the management agent to determine placement of a VM has several purposes, the policy of the agent depends on the purpose. Ghribi et al. [Ghribi2013] used a live migration to increase the number of host machines that can be place in a hibernation state, reducing the power consumption. Akiyama et al. [Akiyama2014] and Vincenzo De Maio et al. [Maio2016] proposed appropriate live migration methods, which also consider the cost of electricity for the migration itself.

Zhaohui $\mathrm{Wu}$ et al. [Wu2016] predicted the temperature of the CPU and used it for the thermal management of the data center, reducing the power consumption required for cooling. Their method can be applied to an environment with dynamic migrations.

Other studies aim to improve the efficiency of live migration itself. C. Li et al. [Li2017] employed an appropriate compression approach according to the network bandwidth, shortening the transmission time for a live migration.

\section{PROPOSAL}

We propose a method to dynamically determine the margins given by the character of the VMs and propose a migration method. Our method deals with the conventional problem in which the CPU utilization fluctuates beyond the fixed margin. As outlined below, this method is roughly divided into two stages: a prediction stage and a migration stage.

1. Prediction Stage

1.1 Calculation of the predicted CPU utilization of a VM

1.2 Calculation of "Stability"

1.3 Decision of the margin

2. Migration Stage

2.1 Detection of overload

2.2 Re-provision of VMs

\subsection{Prediction Stage}

The prediction stage is subdivided into three steps. Overall, the predicted value for CPU utilization at a future time is calculated using the CPU utilization history data. One requirement is that the CPU utilization must be recorded and divided into fixed periods. First, predict the CPU utilization after one period using a linear regression based on the history of CPU utilization. In the conventional method, a fixed margin is given; that is, the expected CPU utilization of $n \%$ is predicted to be the utilization rate of $n+m \%$ when margin is set to $m \%$. In this paper, this margin is set dynamically. To dynamically determine the margin, the stability is calculated for each VM and the margin is determined. After that, the value that includes the calculated margin is used as the predicted value (future CPU utilization).

\subsubsection{Calculation of the Predicted CPU Utilization of Each VM}

The predicted value of CPU utilization is determined based on the linear function derived using the least squares method that is the most popular method to minimize the residual [Farahnakian2013]. The linear function by a linear regression is derived as follows. Using the CPU utilization $y$ with respect to the time $x$, gives

$$
\begin{aligned}
y & =a x+b \\
a & =\frac{S_{x y}}{S_{x}^{2}} \\
& =\frac{\sum_{i=1}^{n}\left(x_{i}-\bar{x}\right)\left(y_{i}-\bar{y}\right)}{\sum_{i=1}^{n}\left(x_{i}-\bar{x}\right)^{2}} \\
b & =\bar{y}-a \bar{x}
\end{aligned}
$$

In this formula, the definition of the character is as follows: 
- $S_{x y}$ is the covariance of $x$ and $y$.

$\cdot S_{x}$ is the standard deviation of $x$.

$\cdot n$ is the number of two-variable data $(x, y)$.

$\cdot x_{i}$ and $y_{i}$ are the individual numerical values.

$\cdot x$ and $y$ are the average values.

Using these formulas, the CPU utilization $u$ at the next time is expressed as

$$
u=a(x+1)+b
$$

\subsubsection{Calculation of Stability}

Stability is derived using the linear function obtained in the previous section. Stability is calculated from the standard deviation of the difference between the predicted value of the CPU utilization $f(x)$ and the actual measurement value $R$ at the time $x$. It is expressed by the formula as follows. $S$ is the standard deviation.

$$
\begin{aligned}
& \text { Delta }_{i}=\text { Utilization }_{i}-(a i+b) \\
& \text { Dispersion }=\frac{1}{n} \sum_{i=1}^{n} \text { Delta }_{i}^{2} \\
& S=\sqrt{\text { Dispersion }}
\end{aligned}
$$

\subsubsection{Decision of the Margin}

Based on the stability derived in the previous section, the margin and the predicted value are determined, and the stability is determined as follows. $C$ is a coefficient, and when the standard deviation is $S$, it is calculated by the function $f(S)$.

$$
\text { Stability }=S \times C
$$

$f(S)$ is a function that is adjusted so that the margin does not become extremely large or small. For example, it is set as follows. When $S$ is small $(<1)$, the coefficient is large. The more $S$ increases, the smaller $f(S)$ becomes. $S$ becomes constant when it exceeds a certain value (10).

$$
c=f(S)=\left\{\begin{array}{c}
5.5(S \leq 1) \\
6-\frac{1}{2} S(1<S<10) \\
1.0(10 \leq S)
\end{array}\right.
$$

\subsection{Migration Stage}

Migration is performed using the predicted values, including the margins obtained in the previous section. The migration stage is divided into two: detection and reallocation.

\subsubsection{Detection of Overload}

When the CPU utilization of the host machine itself increases and reaches a high load state, the VM must be moved to prevent host machine overload. The predicted value of the CPU utilization calculated in the previous section is used to detect this high load state.

A migration is necessary when the total predicted load exceeds $100 \%$ of the host machine capacity. The total predicted load means the sum of the load predictions for all VMs, including the margin. The following procedure is used for the host machine requiring migrations. First, sort the VMs located in the host machine in descending order of load. Then remove VMs from the host machine until the total load is less than or equal to $100 \%$. This is done for all host machines and a list of removed VMs is created. Figure 1 shows the algorithm. 


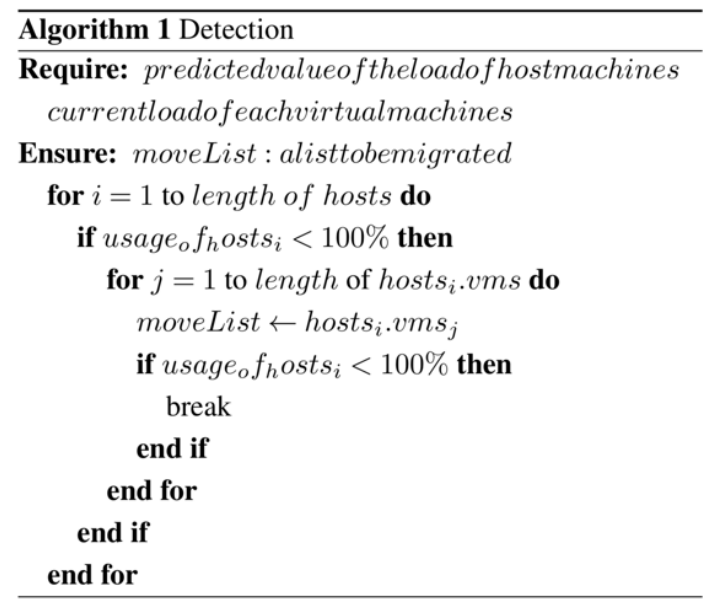

Figure 1. Algorithm to create a migration list

\subsubsection{Re-provision of VMs}

At this stage, the migration destination of the VMs in the generated list is determined. Firstly, sort the VMs in the migration list in descending order of resources consumed. Next, sort the host machines in descending order of resource surplus to create a list of all host machines. The resource surplus of the host machines is expressed by the following equation. The surplus is defined as the capacity of the host machine minus the used resources and stability.

$$
\begin{aligned}
\text { Surplus } & =\text { capacity of host } \\
& - \text { total usage of host } \\
& - \text { total stability of host }
\end{aligned}
$$

Next, remove one VM from the migration list and reallocate it to the host machine with the largest surplus. Then sort the host machines again. Repeat this process until the migration list is empty. If all of the VMs cannot be allocated with the current host machines, then add a new host machine without a VM. This algorithm is similar to the worst-fit algorithm. Figure 2 shows this algorithm.

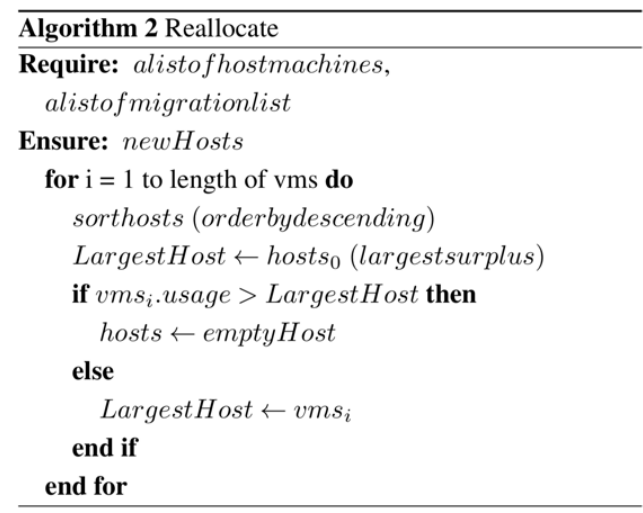

Figure 2. Relocation algorithm

As described above, this operation repeatedly predicts CPU utilization and live migrations. By determining the margin according to each VM and performing migrations, more appropriate resource management can be performed according to the characteristics of the VM. 


\section{EVALUATION}

In this section, we evaluate the proposed method described in Chapter 4. The experiments used a simulated dataset assuming there is a data center where many VMs are allocated. This dataset was acquired as a part of the CoMon project [Kyoung-Soo2012], which has also been used as experimental data in another study [Beloglazov2013]. This dataset contains historical data of the utilization of about 100,000 CPUs which are each used about 288 times on 4394 VMs. Using this data, we compared live migrations for both static margins and dynamic margins. Each experiment was repeated multiple times. Each experiment involved the data from 50 randomly selected VMs.

The evaluation metrics were the total number of times that VMs migrated, the average number of host machines necessary to allocate all VMs, the number of violations in which the CPU utilization of the host machine exceeds $100 \%$, and the total value of the predicted value. The last metric is a measure of how much resources are needed.

Table 1 shows the experimental environment.

Table 1. Experimental environment

\begin{tabular}{|l|l|}
\hline OS & Mac OS (10.1.13) \\
\hline CPU & $3.1 \mathrm{GHz}$ Intel Core i5 \\
\hline RAM & 16GB 2133MHz LPDDR3 \\
\hline Language & Node.js (10.3.0) \\
\hline
\end{tabular}

In the experiment, the CPU of each VM had a single core of $1.7 \mathrm{GHz}, 2.0 \mathrm{GHz}, 2.4 \mathrm{GHz}$, or $3.0 \mathrm{GHz}$ based on the Amazon EC 2 instance type. The CPU of the host machine was changed randomly in the experiment, and the performance was taken as the operating frequency times the number of cores. For example, in the case of four $2.5-\mathrm{GHz}$ cores, the host machine was calculated as one $10-\mathrm{GHz}$ core CPU. Below we introduce the results of several simulations.

\subsection{Experimental Results}

In Experiments 1 and 2, the host machine contained four 2.5-GHz cores. Experiment 2 had a smaller average resource consumption per VM than Experiment 1. Tables 2 and 3 show the results of Experiments 1 and 2 , respectively.

Table 2. Results of Experiment 1

\begin{tabular}{|l|l|l|l|l|}
\hline & Times & Ave. of hosts & Num. of violation & Total value \\
\hline Fixed (conventional) & 52 & 11.97 & 8 & 27795525 \\
\hline Dynamic (this paper) & 50 & 11.97 & 0 & 27942256 \\
\hline
\end{tabular}

Table 3. Results of Experiment 2

\begin{tabular}{|l|l|l|l|l|}
\hline & Times & Ave. of hosts & Num. of violation & Total value \\
\hline Fixed (conventional) & 111 & 8.97 & 8 & 20173017 \\
\hline Dynamic (this paper) & 110 & 8.98 & 3 & 20575375 \\
\hline
\end{tabular}

In Experiment 3, the host machine contained two 2.5-GHz cores. The size of the VM was larger than that for the two previous experiments. Table 4 shows the results.

Table 4. Results of Experiment 3

\begin{tabular}{|l|l|l|l|l|}
\hline & Times & Ave. of hosts & Num. of violation & Total value \\
\hline Fixed (conventional) & 218 & 12.51 & 257 & 13937380 \\
\hline Dynamic (this paper) & 252 & 12.56 & 109 & 14130837 \\
\hline
\end{tabular}

Figures 3-6 shows graphs summarizing the experiments including the remaining two experiments. 


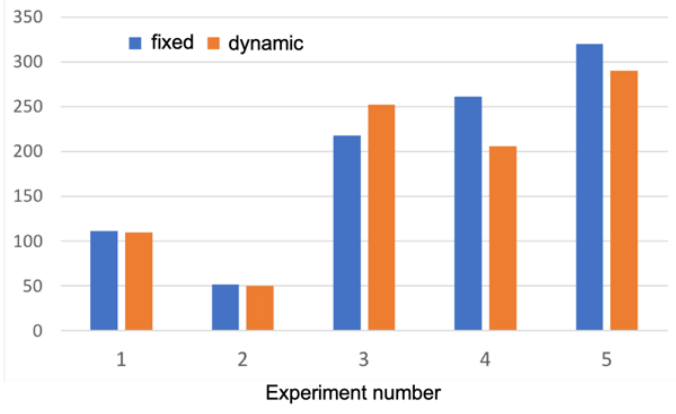

Figure 3. Number of migrations

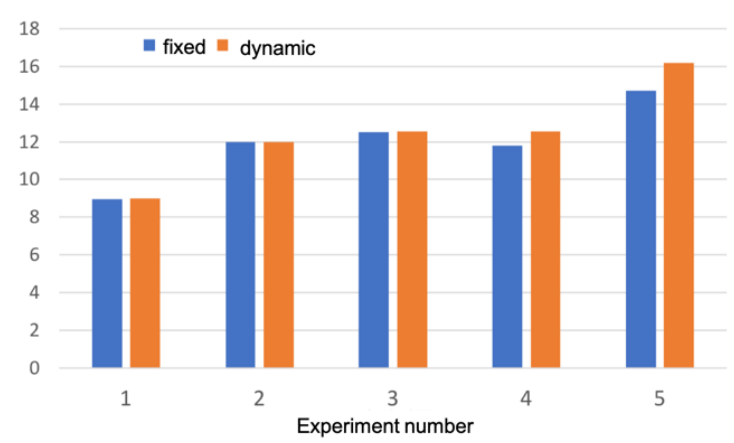

Figure 5. Average number of hosts

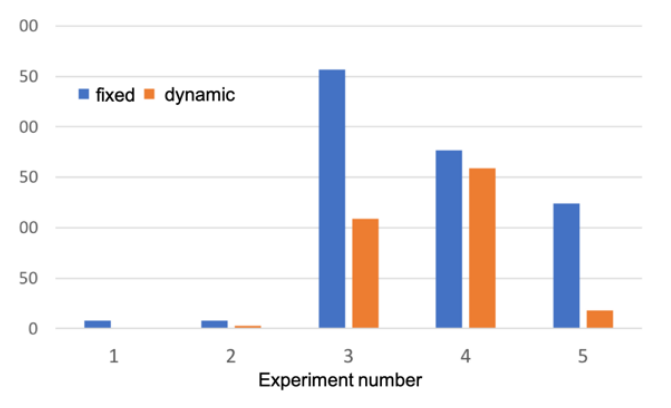

Figure 4. Number of violations

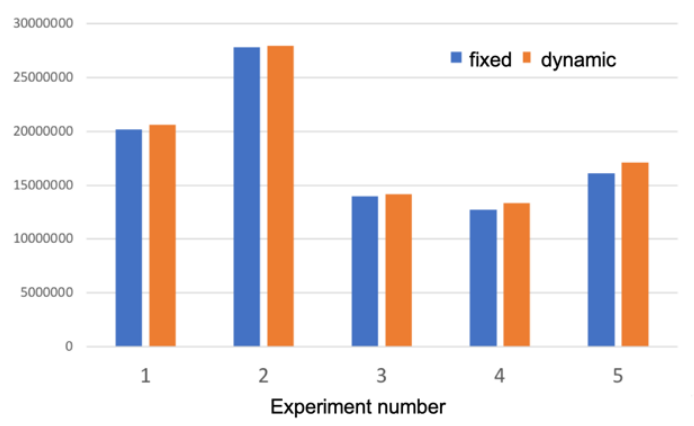

Figure 6. Total allocated resource

\subsection{Discussion}

From the results of Experiments 1 and 2, the number of violations (Fig. 3) is reduced by applying the proposed method compared with the case where the margin is fixed. Additionally, the proposed method has a negligible influence on the number of hosts (Fig. 4) or the total allocated resource (Fig. 5). This can be viewed as achieving the same degree of performance while reducing the number of violations by decreasing the margin when the VM's state is stable but enhancing the margin when the VM's state is unstable. In addition, the increased margin for an unstable VM can be canceled by the reduced margin for a stable VM because the total values are almost the same.

From the results of Experiment 3, the number of migrations increases (Fig. 2), but the number of violations (Fig. 3) decreases. The number of violations of both the fixed margin and the dynamic margin are significantly larger than that of Experiments 1 and 2 because the ratio of resources consumed per VM to the host machine is larger and the degree of influence by the fluctuation of one VM is large.

These experiments including Experiment 4 and 5 demonstrate that the number of violations decreases, while the number of migrations, the average number of hosts, and the total value remain relatively constant. Under certain conditions, the number of violations is reduced by more than $80 \%$ (Experiment 5).

This method gives a small margin when the VM is stable, and a large margin when the VM is unstable. Thus, our method reduces the number of violations compared with the conventional method when the same degree of resource is used. In addition, it can be said that the same efficiency can be demonstrated regardless of the size of the host machine because the total values are similar for the conventional method and our method (Fig. 5).

Web services have load specific patterns. For example, the load is high when user access is temporarily concentrated or periodically high depending on the time of the day. In these situations, our method can be used with a condition suitable for linear regression. If the interval of the collection of CPU utilization becomes short or a shorter period of history is used, our method can handle more drastic changes of CPU utilization. 
Consequently, our method can dynamically change the duration of CPU sampling or the interval by incorporating another method that can detect the period of the load.

\section{CONCLUSION}

In this paper, we first discuss the conventional fixed margin used in CPU utilization predictions of VMs for live migrations. Often the conventional method results in inappropriate resource allocation. To solve this problem, we propose a method to decide the dynamic margin according to the stability, which is calculated by using the difference between the predicted value and the measured value. We also propose a migration method that considers stability.

The evaluation confirms that the number of violations and the number of migrations is reduced compared with the conventional method when the host machine average and the amount of secured resource are similar. The proposed method has the potential to improve the reliability of cloud service with the same resources compared to the conventional method because a cloud service provider can reduce resource consumption by reducing the number of violations. In addition, from the viewpoint of a cloud service user, the cost to maintain web services can be reduced as the need for redundancy is lower.

Although we used a linear regression to predict CPU utilization, it can be predicted by many other methods. Similarly, we only used the CPU utilization to determine migration, but live migrations have other metric such as the amount of memory use and network bandwidth that should be considered. Employing other metric may improve the results of our method. Evaluating the impact of these on our method are future tasks.

\section{REFERENCES}

Abrahao, B. and Zhang, A. Characterizing Application Workloads on CPU Utilization for Utility Computing. Intelligent Enterprise Technologies Laboratory. 2004.

Akiyama,S., Hirofuchi, T. and Honiden, S., Evaluating Impact of Live Migration on Data Center Energy Saving. 2014 IEEE 6th International Conference on Cloud Computing Technology and Science. Singapore, Singapore. 2014.

Beloglazov, A. and Buyya, R., Managing Over-loaded Hosts for Dynamic Consolidation of Virtual Machines in Cloud Data Centers under Quality of Service Constraints, IEEE Transactions on Parallel and Distributed Systems, Vol. 24, No. 7, pp. 1366-1379. 2013.

Farahnakian, F., Liljeberg, P., and Plosila, J., LiR- CUP: Linear regression based CPU usage prediction algorithm for live migration of virtual machines in data centers, Proceedings - 39th Euromicro Conference Series on Software Engineering and Ad-vanced Applications, SEAA 2013, pp. 357-364. 2013.

Ghribi, C., Hadji, M., and Zeghlache, D. Energy efficient VM scheduling for cloud data centers: Exact allocation and migra- tion algorithms, Proceedings - 13th IEEE/ACM International Sym- posium on Cluster, Cloud, and Grid Computing, CCGrid 2013, pp. 671-678 2013.

Hieu, N. T., Di Francesco, M., and Yla-Jaaski, A., Virtual Machine Consolidation with Usage Prediction for Energy-Efficient Cloud Data Centers, Proceedings - 2015 IEEE 8th International Con-ference on Cloud Computing, CLOUD 2015, pp. 750-757. 2015.

KyoungSoo Park, V. P., CoMon Project Retrieved September 20, 2017. from http://comon.cs.princeton.edu/

Li, C., Feng, D., Hua, Y., Xia, W., Qin, L., Huang, Y., and Zhou, Y., BAC: Bandwidth-aware compression for efficient live mi- gration of virtual machines, in IEEE INFOCOM 2017 - IEEE Con-ference on Computer Communications, pp. 1-9. 2017.

Maio, V. D., Kecskemeti, G., and Prodan, R. An Improved Model for Live Migration in Data Centre Simulators, in 2016 16th IEEE/ACM International Symposium on Cluster, Cloud and Grid Computing (CCGrid), pp. 527-530. 2016.

Melhem, S. B., Agarwal, A., Goel, N., and Zaman, M. A Markov-Based Prediction Model for Host Load Detection in Live VM Migration, in 2017 IEEE 5th International Conference on Future Internet of Things and Cloud (FiCloud), pp. 32-38. 2017.

Overcommitting CPU and RAM, Openstack, Retrieved October 28, 2018. from https://docs.openstack.org/archdesign/design-compute/design-compute-overcommit.html, 2018.

Wu, Z., Li, X., Garraghan, P., Jiang, X., Ye, K., and Zomaya, A. Y. Virtual Machine Level Temperature Profiling and Prediction in Cloud Datacenters, in 2016 IEEE 36th International Conference on Distributed Computing Systems (ICDCS), pp. 735-736. 2016. 